\title{
Dynamic presentation of magnified graphical characters on the IBM-compatible computers
}

\author{
VICTOR I. BELOPOLSKY \\ Institute of Psychology RAS, Moscow, Russia \\ and \\ VICTOR E. DUBROVSKY \\ Moscow State University, Moscow, Russia
}

\begin{abstract}
Software that provides extremely smooth gliding and scrolling of magnified characters and pictograms on standard PCs is described. The program was designed as a tool for reading research, as a computer aid for visually impaired readers, and as a subtitle generator for multimedia applications. Change of font, size of characters, speed, color, and direction of movement can be made interactively. The system is useful for reading remediation and for creation of various visual effects.
\end{abstract}

The presentation of continuous text as a one-line horizontally gliding display offers a unique opportunity to remove the vertical dimension from gaze movements. Like the display at Times Square, such gliding displays of text move across the screen horizontally at selected speeds. There have been several attempts to use such displays to investigate search and reading processes (Juola, Ward, \& McNamara, 1982; Legge, Pelli, Rubin, \& Schlester, 1985a; Monk, 1984). The text displays in these studies have been either jerky or smooth, and the texts have been displayed at various rates and with various line widths. Unexpectedly, it has been found that such displays can be read easily and at nearly a normal reading rate. Eye movements recorded during the reading of gliding text show that the average fixation duration is near the average for reading stationary text (Belopolskii, 1989; Buettner, Krischer, \& Meissen, 1985). It has also been shown that the minimum window size that maintains a near normal reading rate is five characters (Legge et al., 1985a). These findings and other psychophysical evidence provided by studies on the reading of gliding text indicate that this technique can be practically applied in such areas as aiding the visually impaired, assisting dyslexic children, and advertising (Krischer, Buettner, \& Meissen, 1991; Krischer, Stein-Arsic, Meissen, \& Zihl, 1985; Legge, Pelli, Rubin, \& Schleske, 1985b).

The studies mentioned above were conducted with the use of expensive specialized video equipment to produce the experimental displays. The ability to produce the same kinds of displays on widely available IBM-PC computers (or compatibles) without special display adapters will

This work was supported by Grant 23/IO-93 from the Russian Institute for Information System Research. Requests for more detailed information about the program and for reprints should be addressed to V. Belopolsky, Institute of Psychology RAS, 13 Yaroslavskaya St., 129366 Moscow, Russia. make this technique more universally available. The commercial software designed to magnify text for editing or reading on computer screens (LPDOS and ZOOMTEXT for the IBM PC and ENLARGE for the Macintosh) has very restricted options for presenting text in the gliding mode. One paper (Krischer et al., 1991) has mentioned the possibility of producing gliding text on PCs, but it did not provide details on implementation. The present paper provides such details.

\section{The Program}

The software system developed by the authors requires an IBM-PC XT or AT (or compatible) equipped with an EGA or VGA display adapter and monitor. The system permits the display of an ordinary ASCII text file in either gliding or scrolling mode. (Scrolling means moving the text display vertically so that the oldest text disappears at the top and the newest appears at the bottom.) The system contains a set of routines that produce the desired display dynamically. The size of the display elements can be varied, depending on the font selected. The speed of the display can be increased beyond the upper threshold of reading while a smooth-appearing movement is maintained.

The display elements are produced in graphics mode by translating the character code in the text to the corresponding pixel pattern contained in the bitmapped font. A special font format is used to speed up the drawing of an individual display element. Font files are maintained on disk and may be loaded prior to or during presentation sessions. Fonts may be designed by the user, or HP (Hewlett-Packard) fonts can be converted for use by a special utility. In addition, display parameters can be placed in a configuration file that can be modified interactively with either the keyboard or a mouse.

The smoothness and speed of display movement are attained by using a combination of methods. First, video 
(A)

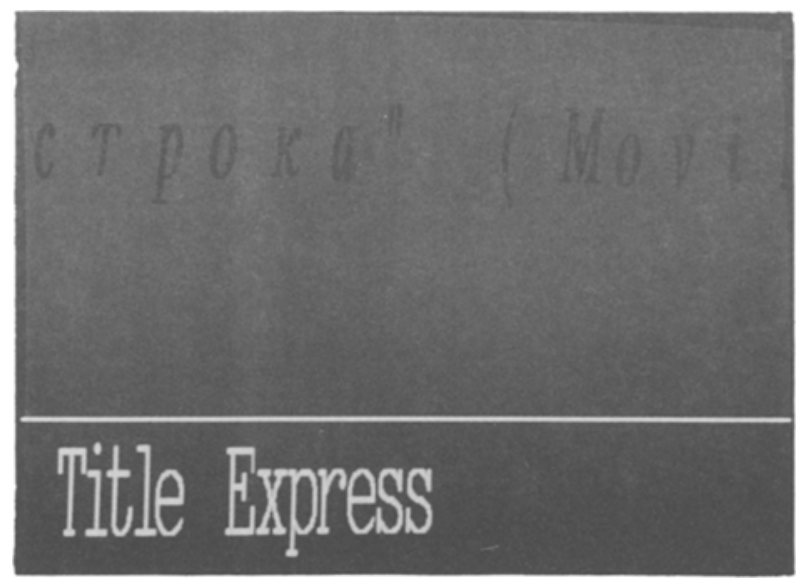

(C)

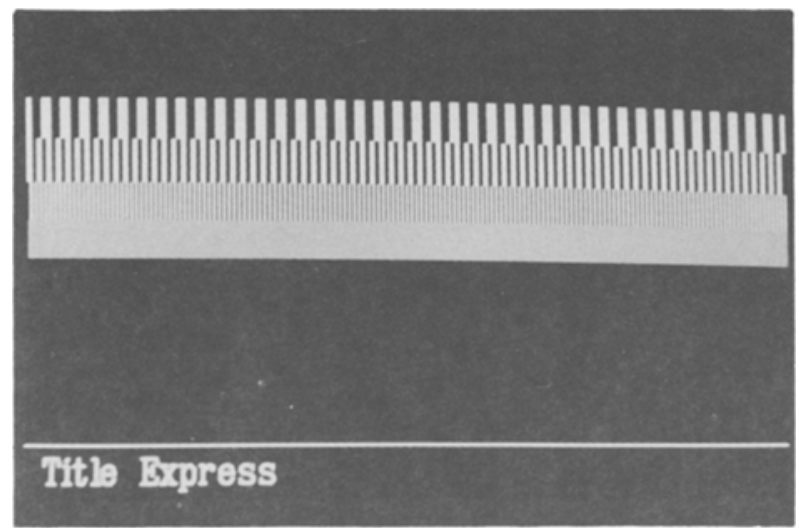

(B)

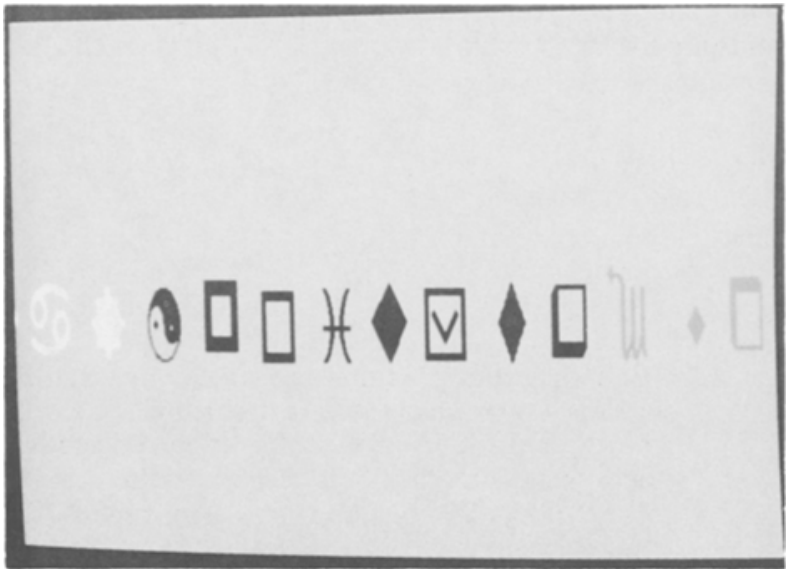

(D)

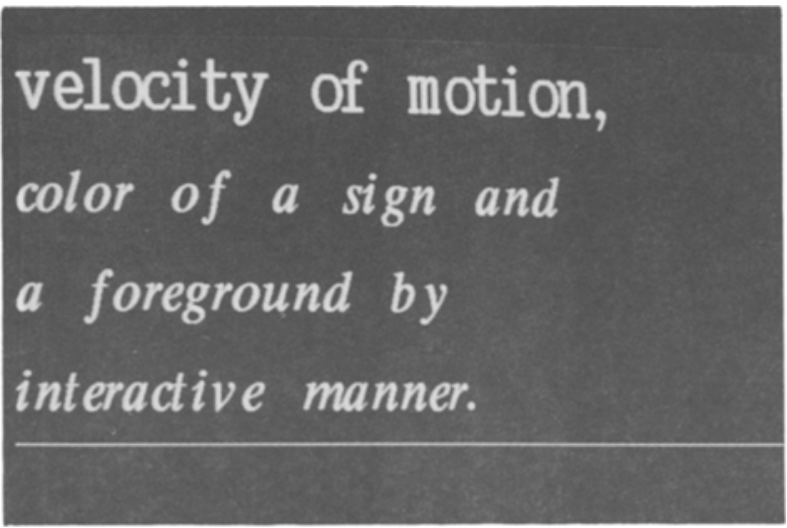

Figure 1. Four pictures of a computer screen that demonstrate different options of the program. Gliding text mode permits presentation of a) text, b) pictograms, and c) pictures. Scrolling text mode (d) has the same options. Screens (a) and (c) show on-line changing of type face and character set (from Cyrilic to Roman). Screen (b) shows the changing of color of pictograms. Background colors are different for these screens. Low parts of the screens are reserved for presentation of static picture, textual, or technical information.

memory is divided into two pages. While one page is being displayed, the other can be modified without "collisions" between the CPU and the display controller. Second, the start address in video memory for the display is also changed. This permits the display to move in the desired direction without requiring a total update of the video memory. Whatever changes are necessary occur during the vertical blanking period and are interrupt driven. Part of the screen can be used to show a stable color picture or to display technical or reference information. Figure 1 shows different screens during execution of the program.

\section{Features and Limitations}

Testing of the software has shown that it is able to support a wide range of character sizes and velocities without loss of smoothness of movement. The time available for modifying the display is limited by the length of the vertical blanking period, which means that there is a tradeoff between the velocity of the text movement and the size of the display element. For example, on a 386/33$\mathrm{MHz}$ PC AT with a VGA display adapter and monitor, at the highest gliding rate and with a display element size of $40 \times 16$ pixels, the time required for a character to move across the screen is $0.83 \mathrm{sec}$. With a display element size 
of $60 \times 48$ pixels, the time required is 2.05 sec. Planned enhancements to the program may reduce these times somewhat. The program uses either block fonts (all elements are the same width) or proportional fonts (each element may be a different width).

\section{Pilot Study}

The rate of silent reading in 10 normal and 4 visually impaired subjects was measured in order to assess the suitability of the system. Character width was varied from 0.8 to $2.4 \mathrm{~cm}$ (16 to 48 pixels). It was found that subjects with normal vision could read the gliding text at a rate of 170 to 230 words per minute (wpm), irrespectively of character width. However, if the angular velocity of the gliding text exceeded $20^{\circ}$ of arc per second, normal readers encountered difficulty. Visually impaired subjects whose visual acuity ranged from $5 \%$ to $20 \%$ were very sensitive to the color, size, and style of the characters, as well as to the background color and the angular velocity of the gliding text. The reading rate for the visually impaired group ranged from 40 to $120 \mathrm{wpm}$. Both groups of subjects assessed the vertically scrolling text as less readable than the horizontally gliding text. In general, the results of this screening agree with earlier findings (see Buettner et al., 1985; Krischer et al., 1985; Legge et al., 1985a, 1985b).

\section{Conclusion}

This software system offers a new approach in the presentation of moving continuous text on a readily available video display. Possible uses include experiments in the fields of information processing and motion perception, a reading aid for the visually impaired, the generation of subtitles, and many others. In particular, it is possible to move gratings with different spatial frequencies across the screen to assess the running visual acuity of patients (Figure 1C). It is also possible to present figures moving smoothly in two dimensions.

\section{REFERENCES}

BELOPOLSKII, V. I. (1989). Eye movement parameters during reading of moving text. Perception, 18, 525 .

Buettner, M., Krischer, C. C., \& Meissen, R. (1985). Characterization of gliding text as a reading stimulus. Bulletin of the Psychonomic Society, 23, 479-482.

Juola, J. F., Ward, N. J., \& McNamara, T. (1982). Visual search and reading of rapid serial presentation of letter strings, words and text. Journal of Experimental Psychology: General, 111, 208-277.

Krischer, C. [C.], Buettner, M., \& Meissen, R. (1991). Reading eye movements for different age levels: The basis to design a tailormade aid for learning to read. In J. van Rensbergen, M. Devijver, \& G. d'Ydewalle (Eds.), In Proceedings of the Sixth European Conference on Eye Movements (pp. 19-20). Belgium: Katholieke Universiteit, Leuven, Laboratory of Experimental Psychology.

Krischer, C. C., Stein-Arsic, M., Meissen, R., \& Zihl, J. (1985). Visual performance and reading capacity of partially sighted persons in a rehabilitation center. American Journal of Optometry \& Physiological Optics, 62, 52-58.

LegGe, G. E., Pelli, D. G., Rubin, G. S., \& Schleske, M. M. (1985a). Psychophysics of reading: I. Normal vision. Vision Research, 25, 239-252.

Legge, G. E., Pelli, D. G., Rubin, G. S., \& Schleske, M. M. (1985b). Psychophysics of reading: II. Low vision. Vision Research, 25, 253-266.

Monk, A. F. (1984). Reading continuous text from a one-line visual display. International Journal of Man-Machine Studies, 21, 269-277. 